LA-UR- 99.2906

Approved for public release; distribution is unlimited.

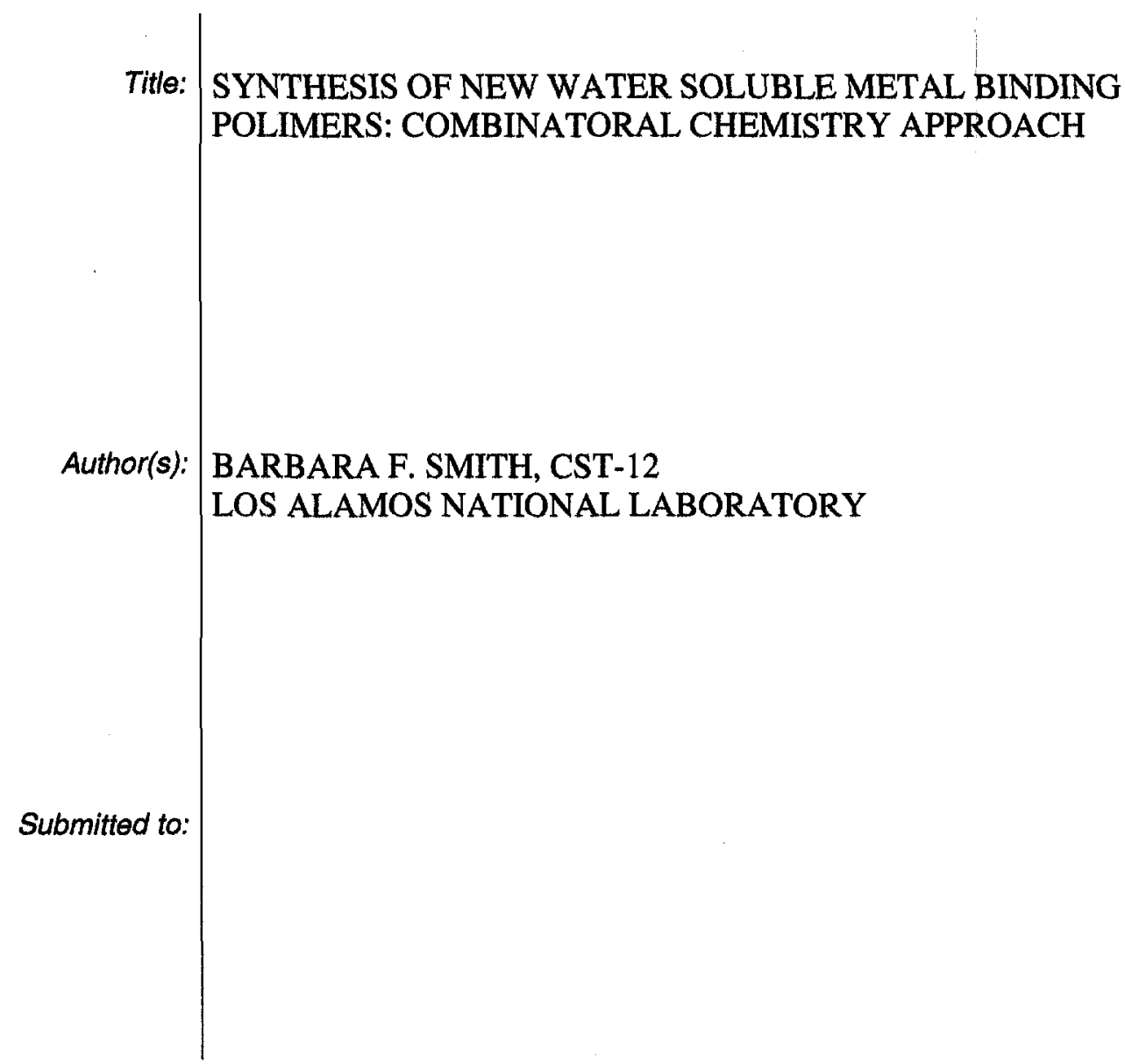

Los Alamos National Laboratory, an affirmative aotion/equal opportunity employer, is operated by the University of California for the U.S. Department of Energy under contract W-7405-ENG-36. By acceptance of this article, the publisher recognizes that the U.S. Government retains a nonexclusive, royalty-free license to publish or reproduce the published form of this contribution, or to allow others to do so, for U.S. Government purposes. Los Alamos National Laboratory requests that the publisher identify this article as work performed under the auspices of the U.S. Department of Energy. Los Alamos National Laboratory strongly supports academic freedom and a researcher's right to publish; as an institution, however, the Laboratory does not endorse the viewpoint of a publication or guarantee its technical correctness. 
Project Title: SYNTHESIS OF NEW WATER-SOLUBLE METAL-BINDING POLYMERS: COMBINATORIAL CHEMISTRY APPROACH

DOE Proposal No. 54724

Publication Date: May 1999

Lead Principal Investigator:

Name: Barbara F. Smith

Institution: Los Alamos National Laboratory, Los Alamos, NM 87544;

Phone: 505-667-2391, e-mail: bfsmith@lanl.gov

Co-Invistigators:

Name: Drs. R. Bryan Miller* and Mark Kurth

Institution: University of California,

Davis, CA;

Phone: 916-752-0963,

e-mail:mjkurth@ucdavis.edu

*deceased

Dr. Samuel Sawan

Lowell, MA

978-934-3680

samsawan@ibm.net: Samuel_Sawan@uml.edu

Graduate Students: Chris Halm, Darin Kizer, Rob Sammelson, Wenjing Zhou, RESEARCH OBJECTIVE

(1) Develop rapid discovery and optimization approaches to new water-soluble chelating polymers for use in Polymer Filtration (PF) systems and (2) evaluate the concept of using water and organic soluble polymers as new "solid" supports for combinatorial synthesis.

\section{RESEARCH PROGRESS AND IMPLICATIONS}

Polymer Filtration (PF), which uses water-soluble metal-binding polymers to sequester metal ions in dilute solution with ultrafiltration (UF) to separate the polymers, is a new technology to selectively remove or recover hazardous and valuable metal ions. Future directions in PF must include rapid development, testing, and characterization of new metal-binding polymers. Thus, we are building upon and adapting the combinatorial chemistry approach developed for rapid molecule generation for the drug industry to the rapid development of new chelating polymers.

We have focused on four areas including the development of: (1) synthetic procedures, (2) small ultrafiltration equipment compatible with organic-and aqueous-based combinatorial synthesis, (3) rapid assay techniques, and (4) polymer characterization techniques.

(1) Synthetic Methods Development: Several classes of materials are being developed: (1) acylpyrazolones, (2) polyisoxazoles, (3) 1,2-aminoalcohols, and (4) pendant-heteroatoms. Recently, we have focused our efforts at obtaining polymers rather than small molecules; and in this effort we have synthesized a wide variety of simple polyacrylamide-containing ligands in order to calibrate our efforts. The polyisoxazole syntheses utilize the 1,3-dipolar cycloaddition of nitrile oxides with alkynes as the dipolarophile. The hope is that these isoxazoles along with other electron donating atoms will have favorable metal binding properties. Our goal is to synthesize a 3,3'-diisoxazole and then tether it with other heterocyles to form tetradentate ligands. Our studies on the construction of pyrazoloisoxazol-(in)e-based heterocycle, utilizing the intramolecular cycloaddition of 1,3-dipole, in turn generated from dipolarophile "tethered" pyrazolo aldehyde have continued. In the acylpyrazolones, we have prepared over 40 derivatives having variation mainly in the side chain at $C-4$. A variety of heterocycles having multidentate ligands such as thiophene, furan, and pyridine have been prepared. 
(2) Small Ultrafiltration Equipment: We have built a unit based on reverse dead-end ultrafiltration. This filtration is faster than a dead-end filter as we are not producing a gel concentration polarization layer on the membrane. We are using centrifugal force as the pressure source to drive the liquid through the membrane. Right now the unit material of construction is a polypropylene housing with a polysulfone membrane. The synthesis can be performed in separate glass or plastic tubes. At the end of the reaction the filtration unit is placed in the tube and then ultrafiltered, driving the solution into the filtration unit from the under side.

3) Rapid Polymer Assaying Techniques: Micro-X-ray fluorescence (MXRMF):: We have been able to optimize the spot drying technique, deal with background noise, optimize thickness and size of the spot, evaluate Mylar base materials, improve sensitivity, and do multi-element analysis. Better sensitivity is realized with lower polymer concentration. We have been successful in detecting $10 \mathrm{ppb}$ concentrations of metal ions in an aqueous test solution. Further improvements with apertures on both the X-ray tube and the detector may increase the method sensitivity.

Radiochemistry: Tests using autoradiography proved to be to slow for a rapid survey approach. Thus we developed a rapid survey technique using direct counting techniques of the ultrafiltration units. We have adapted commercially available, centrifugation driven UF units developed for protein purification to a rapid survey technique of our polymers under various conditions. They use a deadend filtration and have sample volumes from 0.5 to $2 \mathrm{~mL}$. The membranes we use typically have a molecular weight cut off (MWCO) of 10,000 to 100,000 Daltons and are composed of a variety of membrane materials such as cellulose acetate, polysulfone, and fluoropolymers.

(4) Polymer Characterization: The weight average molecular weights, as well as other characteristics such as the second virial coefficients and root-mean-square (RMS) radii of gyration of polyamines and various derivatives, have been determined in solution light scattering studies. The solution dynamics of polyamines and carboxylated and phosphorylated derivatives were studied at $\mathrm{pH}$ 3.3, 7.0 and 10.0. Measurements were made in freshly distilled and de-ionized water as well as in 0.1 $\mathrm{M}, 1 \mathrm{M}$ and 5-M solutions of sodium chloride in water. Molecular weights were calculated from Berry plots. The concentration of sodium chloride leads to an increase in the root mean square radius of gyration, as the polymer chains become more extended and relaxed in a more concentrated salt medium. The apparent molecular weights are observed to decrease in proceeding from acidic to alkaline medium, especially with the phosphorylated polymer. However, the RMS radius of gyration of the carboxyl modified polymer was high at both ends of the $\mathrm{pH}$ scale, i.e., the polymer chains were extended at both the acidic and alkaline $\mathrm{pH}$ ends. The increase in RMS radius of gyration is indicative of increasing solvent-polymer interaction.

\section{PLANNED ACTIVITIES}

Overall activities will include completion of the synthetic methods development and preparing polymers for testing, performing the combinatorial synthesis, testing the polymers on a suite of metals, and characterizing the best candidates. The rapid assay techniques are almost complete, and the polymer characterization direction is towards Cd-NMR. A number of manuscripts are in progress.

\section{INFORMATION ACCESS}

Patent disclosures have been filed to aid in technology transfer

" B. F. Smith, T. W. Robison and G. D. Jarvinen,"Water-Soluble Metal-Binding Polymers with Ultrafiltration: A Technology for the Removal, Concentration, and Recovery of Metal Ions from Aqueous Streams", ACS Symposium Series volume, Advances in Metal Ion Separation and Preconcentration, R. Rogers, A. Bond and M. Dietz eds, Chap. 20, pp 294-330, 1998.

Lisa Colletti, George Havrilla, "Trace Element Detection with Micro-X-Ray Fluorescence", Advances in X-Ray Analysis, Vol 44, 1999. 
Darin E. Kizer, R. Bryan Miller, and Mark J. Kurth. Fused Pyrazolo Heterocycles: Intramolecular [3+2]-Nitrile Oxide Cycloadditions Applied to Syntheses of Pyrazolo[3,4-g][2,1]dihydrobenzoisoxazol(in)es. Tetrahedron Letters, 40: 3535-38, 1999.

In-Prep Robert E. Sammelson, R. Bryan Miller, and Mark J. Kurth. The Synthesis of Tetraheterocyclic Polyisoxazole Chelating Ligands Using the Intermolecular 1,3-Dipolar Cycladdition Reaction. Journal of Organic Chemistry. 